\title{
Utilizando Mapas Cognitivos para Compreender Interorganizações: O Mapeamento nos Postos de Emissão de Identidade.
}

\author{
Emiliana da Costa Vargens*
}

\begin{abstract}
Resumo: Este trabalho faz a análise do comportamento das pessoas, através da técnica de mapas cognitivos, em organizações que estão inter-relacionadas por participarem de uma rede de serviços de identificação. Os mapeamentos cognitivos foram realizados no posto central de atendimento do Instituto Pedro Mello - IPM e em um dos postos de atendimento do Serviço de Atendimento ao Cidadão - SAC. Com o mapeamento foi possível identificar conceitos e crenças compartilhadas por funcionários de cada um dos postos, para então analisar a relação entre os funcionários do IPM e do posto do SAC ao estarem trabalhando de maneira interdependente. Além de buscar analisar o comportamento das pessoas nas organizações em questão, este trabalho de pesquisa tem ainda como objetivo demonstrar como a construção de mapas cognitivos pode ser útil para tal análise.
\end{abstract}

Palavras-chave: cognição, mapeamento cognitivo, comportamento organizacional, interorganizações.

Abstract: This research work suggest an analyse of human behavior, through cognitive map technic, in organizations that are envolved in the same network. The research was accomplished in the central post of Intituto Pedro Mello, organization responsable of civil identification in state of Bahia, and in one of attendind post of Serviço de atendimento ao Cidadão - SAC. Through the mapping, it was posible to identify shered meanings and belives of groups servants of each post. Than, it was posible to analyse the relationship between these two groups in the network Beyond trying to understand human behavior, this work intend to demonstrate how cognitive mapps can be useful to organization studies.

Key-words: cognition, cognitive mapping, organizational behavior, interorganizations.

\section{Introdução}

Os postos do Serviço de Atendimento ao Cidadão - SAC são reconhecidos como diferenciais em relação aos demais serviços de atendimento ao público da Bahia. Tratando das agênciasi de identificação do SAC, especificamente, e dos demais postos de identificação do Instituto Pedro Mello, pode-se dizer que eles operam em diferentes padrões de atendimento. $\mathrm{O}$ interesse em identificar e comparar conceitos relacionados a atividade de emissão de carteira de identidade pelo Instituto Pedro Mello e pelo Serviço de Atendimento ao Cidadão - SAC através da elaboração de mapas cognitivos parte de um

\footnotetext{
* Mestre em Administração pelo NPGA/EAUFBA
} 
estudo, no qual tenta-se compreender a relação entre os atores da rede de serviços que envolve a participação da Secretaria de Administração do Estado da Bahia, do Instituto Pedro Mello e dos Postos de Atendimento do SAC.

Para melhor compreender o objetivo deste trabalho é preciso, inicialmente, visualizar a configuração dessa rede de serviços. O SAC é formado através de parcerias entre a SAEB e outros órgãos municipais, estaduais, federais e até mesmo da iniciativa privada, sendo que de cada uma dessas parcerias resulta em agências de serviços nos postos de atendimento do SAC espalhados pelo Estado da Bahia.

Nesse caso, vamos tratar do Instituto Pedro Mello, organização integrante da Secretaria de Segurança Pública da Bahia, e da sua respectiva agência para emissão de documento de identidade no posto do SAC/Barra. Esta agência, bem como as agências dos demais postos, está sob controle operacional da SAEB, sempre representada pela administração de cada posto, e sob controle técnico do Instituto Pedro Mello, através de alguns dos seus funcionários que supervisionam as tarefas ali realizadas e que estão em contato direto com o Instituto.

Sabendo-se que o Instituto Pedro Mello é responsável pela coordenação da emissão de carteiras de identidade pelos postos de atendimento do SAC, pretende-se aqui mapear conceitos relacionados à mesma atividade de atendimento para confecção de carteiras nos seguintes postos:

- Posto Central do prédio do Instituto Pedro Mello, no qual a atividade é realizada por funcionários públicos e concursados;

- Posto do SAC/Barra, no qual a atividade é realizada por funcionários terceirizados, da empresa PostData, e contratados pela Secretaria de Administração do Estado da Bahia treinados pela SAEB para prestar esse atendimento.

Acredita-se que a comparação entre os dois mapas e o levantamento de possíveis diferentes conceitos sobre a mesma atividade, poderá contribuir para avaliar $\mathrm{o}$ relacionamento entre essas duas organizações e compreender o funcionamento do SAC como um organização em rede.

Como estrutura desse trabalho, cabe, inicialmente, a revisão de alguns aspectos conceituais sobre a relação entre cognição e organização, conceitualização de mapas cognitivos e a sua utilidade como ferramenta de pesquisa organizacional. Em seguida pretende-se descrever a metodologia adotada para a confecção dos mapas, para então fazer a análise dos mesmos. A título de conclusão será averiguada a relevância das informações representadas nos mapas obtidos nesta atividade para a compreensão das relações interorganizacionais entre as entidades em questão.

\section{Aspectos Conceituais Sobre Mapas Cognitivos}

\section{Cognição e organização}

Ao tratar de cognição e organização, BASTOS (2000) diz que a natureza dos estudos e conceitos sobre este tema pautam-se em uma concepção da relação entre teoria e prática, que busca oferecer àqueles envolvidos com a construção das organizações, uma abordagem compreensiva dos processos e fenômenos que definem o seu dia a dia, destacando o papel que os indivíduos singulares e em interação desempenham. $O$ autor argumenta que a interface entre os estudos cognitivos e organizacionais fornece uma nova 
linguagem e desloca a atenção para novos focos ou fenômenos que afetam o desempenho de indivíduos, grupos e organizações. Daí resultam novas concepções sobre o comportamento humano de natureza descritiva.

Os estudos no campo cognitivo orientados para organizações ao longo do último século concentram-se em duas grandes matrizes (BASTOS, 2000). A primeira trata a organização como um conjunto de indivíduos em interação e enfatiza mais o papel do indivíduo na construção da organização, do que no papel da organização como determinante de processos individuais. A segunda matriz reifica o conceito de organização, considerando que esta tem uma existência própria independente das pessoas e de suas atividades. Essa matriz considera que as organizações tem o poder de moldar o comportamento e as ações individuais. Situando-se na primeira matriz apontada por BASTOS (2000), encontra-se a cognição social, que este mesmo autor define como um conjunto de conceitos e metodologias que se ocupa da análise dos processos através dos quais os indivíduos percebem, estruturam e usam o conhecimento sobre si, sobre os outros e sobre o mundo.

PORAC et all (1996) salientam que, como a pesquisa em geral para o conhecimento e percepção, o construtivismo traz uma importante contribuição, na medida em que rompe com a distinção entre indivíduo e ambiente, considerando que o ambiente é ao menos um contingente parcial no indivíduo ou grupo que o percebe, um contingente que tornam verdadeiras afirmações relativas a uma comunidade de crenças. De acordo com esses autores isso envolve sistemas de significados, parcialmente compartilhados e parcialmente idiossincráticos predominantes no ponto entre decisão e ação.

Os estudos cognitivos envolvem processamento simbólico que permite às pessoas representar o mundo em termos de um modelo mental, no qual as operações mentais ${ }^{\mathrm{ii}}$ consistem em manipular esses símbolos, ao qual se pode aplicar regras (conscientes ou inconscientes) para fazer inferências (BASTOS, 2000).

Considerando que organizações são produtos de ações individuais e coletivas, e que sua dinâmica e seus processos entrelaçam-se com processos e dinâmicas de indivíduos e de grupos em um espaço e tempo que delimitam e circunscrevem suas interações (BASTOS,2000), o trabalho de PORAC et all (1996) reafirma a tendência dos estudos cognitivos, que deixam de ser fenômenos intrapsíquicos para uma abordagem de processos humanos individuais e coletivos, já que estes autores tratam mais de significados, representações e sentidos, do que cálculos e escolhas que caracterizavam os estudos cognitivos quando para se entender o sentido de mente usava-se a metáfora do computador $^{\text {iii }}$. Ou seja, focalizam muito mais no contexto social do que nos processos mentais individuais, permitindo, com isso, diversos estudos sobre cognição dentro das organizações.

De acordo com BASTOS (2000), os estudos cognitivistas estão orientados para fornecer a indivíduos e gestores ferramentas para entender os processos e sobre eles intervir. Esses estudos, portanto, abordam questões que vão desde as percepções individuais sobre eventos e chegam até os processos pelos quais o ambiente é interpretado e percebido, e como essas percepções influem na formulação de planos, projetos e políticas. Os estudos sobre teorias implícitas de organização apontam para a associação das abordagens cognitivas com assuntos organizacionais mais amplos, tais como design e estrutura organizacionais (DOWNEY e BRIEF, 1996).

Revisando o estado da arte no campo da cognição e organização, BASTOS (2000) afirma que é forte, nesta área, o pressuposto de que a cognição individual produz 
comportamento organizacional, o que aponta para uma visão dos indivíduos como agentes causais e dos processos coletivos como o somatório de processos individuais. Sendo assim, esse mesmo autor ressalta que os mapas cognitivos têm sido utilizados em diversos domínios organizacionais, para identificar estruturas de conhecimento que guiam a percepção, julgamento e decisões, tanto em nível de indivíduos, como de grupo, da organização ou de grupos de organizações. BASTOS (2000) argumenta que o mapeamento cognitivo, por ser uma ferramenta interativa que busca clarificar problemas complexos e facilitar o processo grupal de encontrar soluções, é reconhecido como uma das estratégias metodológicas mais adequadas à perspectiva cognitivista dos processos organizacionais.

\section{Mapas cognitivos como ferramenta de pesquisa organizacional}

Os mapas cognitivos são amplamente utilizados nas pesquisas sobre cognição social, que está situada na primeira matriz apontada por BASTOS (2000), e que é definida por este mesmo autor como um conjunto de conceitos e metodologias que se ocupa da análise dos processos através dos quais os indivíduos percebem, estruturam e usam o conhecimento sobre si, sobre os outros e sobre o mundo.

BASTOS (2000) conceitua mapas cognitivos como "representações, schemas ou modelos mentais construídos pelos indivíduos, a partir das suas interações $e$ aprendizagens em um domínio específico do seu ambiente, e que cumprem a função de dar sentido à realidade e permiti-lhe lidar com os problemas e desafios que esta lhe coloca".

De acordo com este autor, para melhor compreender o conceito de mapa cognitivo, deve-se considerar que os mapas não podem ser vistos como representações estáticas, pois as construções que fazemos da realidade estão sujeitas a novas informações de forma que permitam o ajustamento do indivíduo ao seu meio ambiente. BASTOS (2000) argumenta ainda que, como uma representação da realidade, os mapas envolvem simplificação e fornecem uma imagem aproximada desta realidade. O processo de mapear é impreciso, já que este é um processo de natureza inferencial, e a realidade está sempre em mudança.

LAUKKANEN (1994) realizou uma pesquisa sobre cognição organizacional gerencial através de um estudo de caso, no qual são comparadas estruturas cognitivas de gerentes em duas indústrias para que, então, fossem mapeados conceitos bases e crenças causais que fornecessem o entendimento de como estes gerente pensam e agem. Nesse estudo o autor chama atenção para $o$ fato de que o mapeamento cognitivo nas organizações é influenciado por várias tendências cognitivas humanas gerais. Este autor diz que as pessoas baseiam-se freqüentemente em percepções e inferências; a inteligência e o pensamento humano são orientados para serem usados para sobreviver ou para outros fins práticos; a memória das pessoas não representa um sistema de estrutura linear e o seu acesso e processamento está associado a estímulos e ligado por atividades cognitivas; e, ainda, vários fatores contextuais, como o fenômeno de pensamento do grupo, influenciam os acontecimentos. De acordo com LAUKKANEN (1994), estas são questões complexas e difíceis de generalizar, porém é certo que existe a necessidade de explorar aqueles elementos e aspectos cognitivos dos gerentes que aparecem quando a ação corporativa é contemplada e decidida. 
Ao estudar métodos de análise de discurso, POESI (1999) trata de mapas cognitivos como métodos estruturados para coletar discursos ou representações que têm por objetivo gerar diretamente a representação de um sujeito no que diz respeito a um problema ou tema que interessa ao pesquisador.

BOUGON (1983) diz que a percepção, conceitualização e comportamentos de uma pessoa surgem da interação da sua experiência com seus esquemas. Considerando que conceitos são noções por trás de palavras e que esquema pode reconstruir um padrão de conceitos arquivados, BOUGON (1983) argumenta que, se os conceitos na estrutura conceitual de uma pessoa são rotulados por palavras, nós podemos explorá-los.

Segundo BOUGON (1983), mapeando a estrutura conceitual de um ator em uma entidade social, estamos mapeando o que significa para ele aquela entidade e a experiência daquela entidade. $\mathrm{O}$ autor diz que um mapa cognitivo contém a representação do eu e a representação do ambiente de um ator social, assim como o padrão do relacionamento entre elas.

BASTOS (2000) refere-se ao mapeamento cognitivo entendido como o conjunto de técnicas ou ferramentas de pesquisa voltadas para identificar os elementos que integram esses mapas ou modelos constituídos pelos indivíduos e partilhados por outros. Essas técnicas envolvem formas de retratar graficamente as crenças subjetivas acessadas, dandolhes um tratamento especial que facilite a visualização das relações significativas identificadas.

Este autor aponta para o interesse crescente dos pesquisadores na utilização de mapas cognitivos para compreender os elos cognitivos envolvidos nas decisões e ações organizacionais, bem como na sua utilização como ferramenta para diagnosticar e aprimorar os processos de gestão, considerando que as cognições dos atores são aspectos fundamentais para se compreender a dinâmica dos processos coletivos ou organizativos. BOUGON et all (1977), em uma pesquisa com uma orquestra de jazz, mostrou que é possível descrever as estruturas de conhecimento dos grupos organizacionais.

HUFF (1990) argumenta que alguns cientistas cognitivistas são fascinados demais por um modelo computacional da atividade mental, outros acham que todos os mapas levam a uma interpretação não acurada da cognição, e outros perturbam-se pela simplificação dos modelos mentais. Segundo este autor, o posicionamento conservador trata o mapa como uma ferramenta sujeita às mesmas padronizações que governam o uso de todas as ferramentas de pesquisa. No entanto, HUFF (1990) acredita que os mapas mentais podem ser mais do que um instrumento metodológico, e que através deles podemos extrair informações fiéis às características essenciais do próprio pensamento. $\mathrm{O}$ autor diz ainda que o mapa mental é um conhecimento que os próprios sujeitos utilizam.

De acordo com BASTOS (2000), os mapas podem ser construídos a partir de dados brutos de diferentes naturezas, oriundos de fontes documentais ou de relatos verbais produzidos em condições planejadas para a finalidade que o mapeamento objetiva.

HUFF (1990) e BOUGON (1983) destacam que a diversidade dos possíveis métodos de mapeamento também vem de uma diversidade de relações potenciais entre os elementos cognitivos. Podem ser identificados aqui simples associações (A me lembra B), grau de similaridade (A e B são diferentes), valor relativo (A é mais importante que B) e vínculo causal (A causa B). Ou então pode-se mapear argumentações (uma vez que A é verdadeiro, então $B$ não é verdadeiro), enfocar escolhas (uma vez que $A$, então nós devemos fazer B) e fazer inferências a partir de um texto (uma vez que A e B são mencionados, o informante deve ser influenciado por $\mathrm{C}$ ). 
BASTOS (2000) destaca três tipos de mapas cognitivos:

- Mapas de identidade: apóiam-se numa análise de conteúdo para identificar conceitos e temas centrais nos discursos enunciados pelos indivíduos, apontam as principais características do terreno cognitivo e as atividades envolvidas na sua construção são básicas para todos os demais tipos de mapas.

- Mapas de categorização: buscam descrever os schemas utilizados por gestores ao agruparem eventos e situações com base em suas semelhanças e diferenças, tendo acesso ao sistema de categorias de pensamento utilizado e a dimensão de hierarquia que existe entre esses conceitos.

- Mapas causais: são os mais difundidos nos estudos gerenciais e fornecem uma compreensão dos vínculos que indivíduos estabelecem entre ações e resultados ao longo do tempo.

\section{O mapeamento cognitivo em interorganizações}

Ao analisarem as relações que emergem da interação entre os diversos atores de uma rede, HARD e PHILLIPS (1998) utilizam o conceito de domínio interorganizacional, que emerge quando diferentes organizações se percebem conectadas por questões comuns. $\mathrm{O}$ domínio interorganizacional não é uma estrutura predeterminada, mas um processo de construção social e criação de significado.

Ao constituir uma rede, os interesses de uma organização e do sistema devem ser mutuamente adaptados um ao outro e os atores devem compartilhar uma visão a respeito das questões e dos participantes que constituem o domínio interorganizacional. Esta visão compartilhada cria uma identidade para o domínio, e mutuamente concorda com direções e fronteiras, que podem ser reconhecidas e adotadas como uma estrutura permanente. Porém, se os atores não estão em concordância, então cada qual vai querer usar o seu poder para influenciar o domínio a seu favor. Dessa forma, o desenvolvimento de um domínio interorganizacional é um processo de construção social que permite que os parceiros se comuniquem, sejam identificados e legitimados, e reconheçam os problemas que os confrontam. Porém, se os atores não estão em concordância a respeito dos objetivos da constituição da rede, da maneira como devem ser realizadas as atividades, ou como devem se estabelecer as relações de troca entre eles, então cada qual vai querer usar o seu poder para influenciar a construção do domínio a seu favor, de maneira que possa obter a maior vantagem deste.

O fato é que as organizações, ao resolverem agir em conjunto, estarão apostando no domínio interorganizacional que, como já foi dito, é socialmente construído. Dessa forma, haverá um esforço de cada um dos atores em fazer todo o possível para assegurar que ele seja construído de acordo com seus interesses. Devemos considerar também que a habilidade de participar de uma construção social - de criar significados - é diferencialmente distribuída entre os atores e, portanto, aqueles com maior poder terão maior influência no domínio interorganizacional.

Parece de extrema relevância a construção de mapas para auxiliar a compreensão da construção de um domínio interorganizacional, já que este se constitui de significados compartilhados ou não pelos diferentes atores envolvidos. Através do mapeamento cognitivo será possível identificar conceitos relativos a membros isolados ou grupos de 
indivíduos nas organizações. Tais conceitos, por sua vez, poderão ser úteis para compreender o comportamento dos indivíduos no sentido de cooperar ou bloquear a ação do outro dentro de uma rede.

Dessa forma, os mapas que aqui foram elaborados irão contribuir para a análise de conceitos compartilhados ou não pelos membros da rede que promove a emissão de documentos de identidade pelo SAC/Barra, fornecendo, com isso, uma visão do domínio socialmente construído por esses autores, e uma previsão de como estes atores imaginam que podem influenciá-lo.

\section{Procedimentos}

Para objetivos deste trabalho foram feitos dois mapas cognitivos, o primeiro através de informações obtidas no posto central de atendimento situado no Instituto Pedro Mello, e o segundo da na agência situada no SAC/Barra. O mapeamento foi feito através de relatos verbais de um grupo de funcionários de cada uma dessas organizações, obtidos com entrevistas. Todas os entrevistados têm a mesma função de atender ao público para emissão de carteira de identidade.

Durante as entrevistas não houve intervenção do entrevistador e para todos os entrevistados foram feitas, individualmente, as mesmas perguntas. A entrevista foi orientada para obter opiniões e descrições a respeito da função desempenhada pelo funcionário, das pessoas que o procura para tirar o documento, das características que o funcionário deve ter para exercer tal função, e de como o funcionário deve se comportar numa situação de conflito com as pessoas que são atendidas.

Os mapas utilizados neste trabalho são mapas de identidade, nos quais são identificados conceitos que os entrevistados formulam a respeito da atividade que executam, de si mesmos nessa atividade e das pessoas a quem atendem. Esses mapas apresentam também o grau de importância desses conceitos e como os mesmos estão associados.

Os mapas apresentam três campos conceituais: um referente ao funcionário, outro referente ao público, e um terceiro referente à atividade, buscando, com isso, analisar como as pessoas nessas organizações percebem a atividade que realizam, o público a quem atendem e a si mesmos no contexto organizacional. A posição dos campos conceituais reflete a idéia de hierarquia entre os conceitos.

\section{Mapeamento no Instituto Pedro Mello}

As entrevistas foram realizadas no posto central do Instituto Pedro Mello com três dos seus atendentes, que são funcionários públicos e, através de concurso público receberam o cargo de Perito Técnico de Polícia.

\section{Mapa 1}


O campo relacionado ao funcionário tem como principal conceito o "perito técnico de polícia", pois é como os funcionários mais se identificam. Além deste termo aparecer mais de uma vez no texto, a atividade é descrita com minuciosidades que expressam a preocupação que o perito deve ter ao realizá-la. Pelo mesmo motivo, no campo referente a atividade, temos como principal conceito "identificar pessoas" e para o público temos "pessoas comuns", que é reforçado pelas expressões "irmão", "igual a mim" e "ser humano", como aparecem no texto. Então, "peritos técnicos de polícia identificam pessoas comuns" seria a idéia principal que os entrevistados têm a respeito do trabalho que executam, e por isso estão em destaque.

Outros conceitos podem ser identificados e associados. O funcionário deve ser "calmo, paciente e educado" para exercer uma atividade de "atendimento ao público", público este de pessoas que "têm problemas, chegam estressadas e precisam de ajuda". Por outro lado, o funcionário também deve ser "inflexível em relação aos procedimentos técnicos", já que se trata de uma "atividade de grande responsabilidade", embora o público ache que "a atividade de identificar é muito simples". Esses conceitos são reforçados pelo fato de que os funcionários estão sempre tentando "explicar o serviço", como eles mesmos dizem mais de uma vez. Vale observar que a expressão "inflexível em relação aos procedimentos técnicos" é a única que não foi extraída do texto, porém pode ser concluído que os funcionários pensam assim, não só pela forma minuciosa como é descrita a atividade de identificar, como também pelos problemas relatados que impedem a identificação.

Os conceitos relacionados ao funcionário estão acima porque são considerados os de maior peso. Segundo os depoimentos, podemos deduzir que eles são o foco principal da atividade, que não pode ser realizada sem eles, pois o público precisa que eles o identifiquem. Esta conclusão pode derivar de vários momentos das entrevistas. Por exemplo, quando foi perguntado a respeito da importância do trabalho, o funcionário respondeu que seus conhecimentos são muito importantes, e quando trata-se de definir a função, respondeu, de forma imperativa, que identifica pessoas, como se só o "perito técnico de polícia" dispusesse desse poder. 
MAPA 1: Posto Central do Instituto Pedro Mello

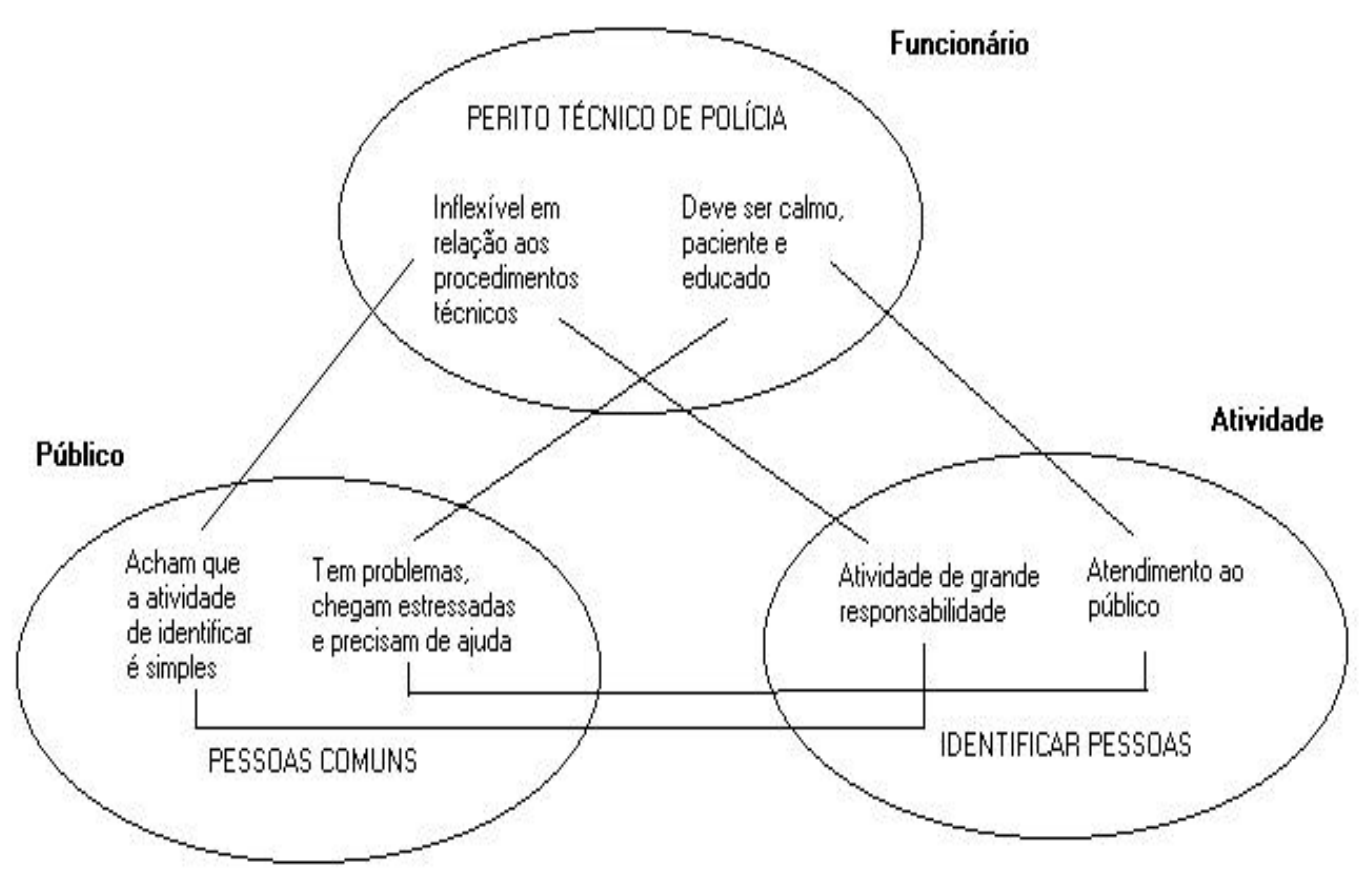

\section{Mapeamento no Posto do SAC/Barra}

As entrevistas foram realizadas na agência de emissão de documento de identidade do posto do SAC/Barra com três dos seus atendentes, que são funcionários terceirizados contratados pela Secretaria de Administração do Estado - SAEB. Esses funcionários estão sob a supervisão de técnicos do Instituto Pedro Mello e também da SAEB, representada pela administração do posto. Eles recebem treinamento tanto no Instituto, quanto na SAEB.

\section{Mapa 2}

No campo referente à atividade temos como principal conceito "emissão de documento de identidade". Esse é o campo mais parecido com o mapa anterior, porém os funcionários aqui não dizem que identificam pessoas e sim que trabalham na emissão de documento de identidade. O público é formado, sobretudo, por "cidadãos" e o funcionário tem como principal conceito "atendente", pois é como mais se identificam.

Outros conceitos podem ser identificados e associados. A atividade também é de "atendimento ao público", que também são "clientes que querem comprar um produto", e o funcionário, portanto, precisa ser "paciente, calmo, simpático e deve saber ouvir". Por outro lado, esta atividade trata-se da "confecção de um documento de grande 
importância", pois o público é formado por cidadãos que "precisam da identidade para ser gente" e, portanto, o funcionário "deve estar atento para não errar".

MAPA 2: Posto SAC/Shopping Barra

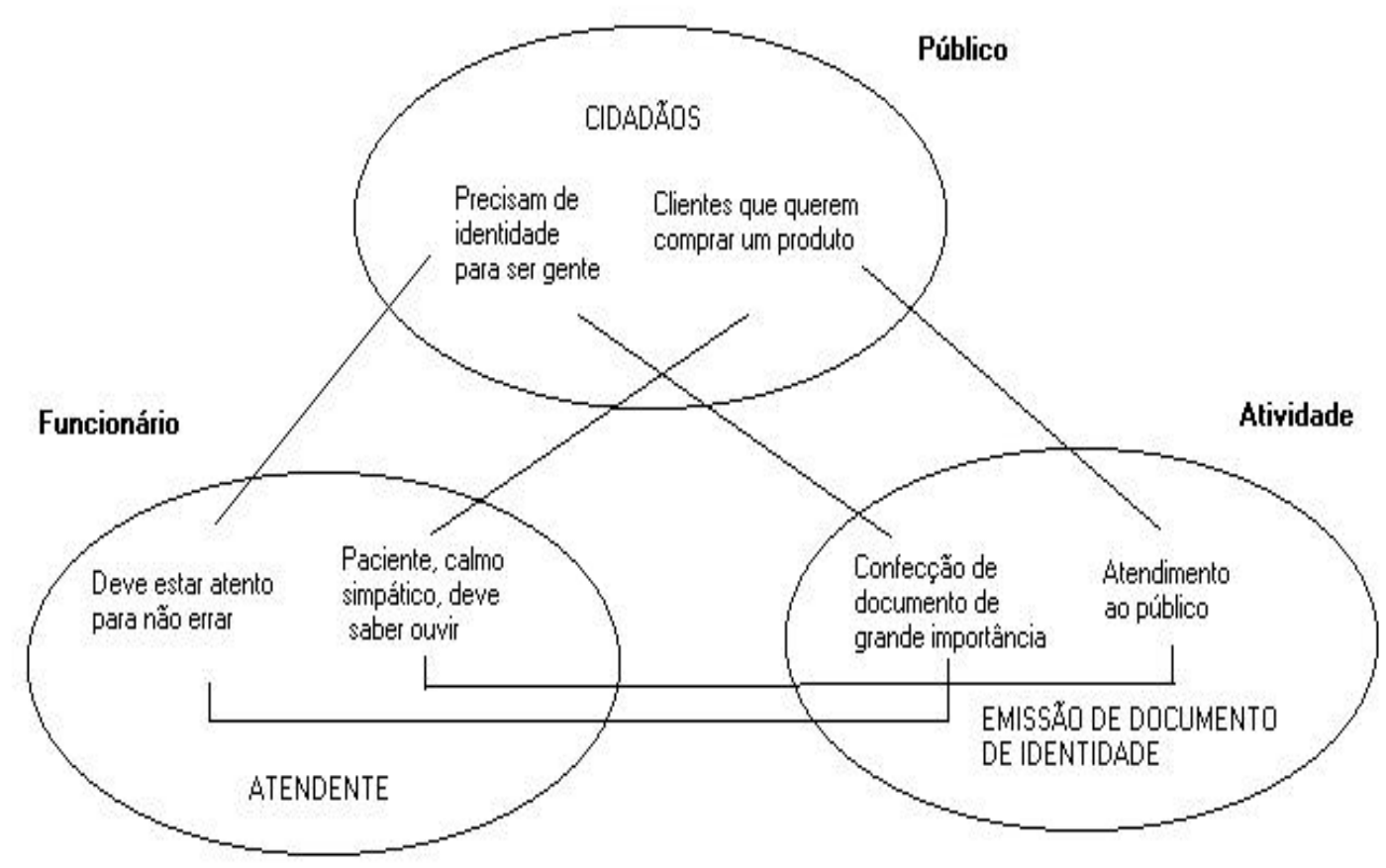

Os conceitos relacionados ao público estão acima porque são considerados os de maior peso, pois o cidadão é o foco. Aqui, o funcionário chega a dizer que é de fundamental importância que as pessoas venham, pois sem elas não existe trabalho. E quando perguntamos sobre a importância do trabalho a resposta é que é excelente para a comunidade, ou que este é um documento que identifica o cidadão.

\section{Conclusões}

Mesmo sabendo que os mapas causais são os mais utilizados nos Estudos Organizacionais, especialmente para fazer comparações entre organizações, a escolha desse tipo de mapa cognitivo se justifica por estes mapas serem fruto do primeiro contato com as pessoas entrevistas. Portanto, antes de reconhecer ações e as relações de causalidade entre elas, o interesse é identificar conceitos, verificando quais são os que mais aparecem, os mais importantes e se estão relacionados entre si. Além do mais, é sabido que o mapa de identidade é o ponto de partida para os demais e, portanto, este mapa seria útil para futuros trabalhos, nos quais outras entrevistas poderiam ser feitas a 
partir das informações obtidas com os mapas de identidade aqui desenvolvidos, e poderiam ajudar na construção de mapas causais.

Ainda a respeito da confecção dos mapas, é importante lembrar que nem todos os conceitos de cada entrevista, individualmente, estão expressos nos mapas, pois já que trata-se de um mapa para cada grupo de três pessoas, procuroutse expressar aqueles conceitos que foram ditos e foram, explícita ou implicitamente, reforçados.

A análise dos dois mapas deixa claro a diferença do modo de pensar dos dois grupos de funcionários a respeito do seu papel, das atividades que executam e das pessoas a quem servem. Isso pode indicar aonde devemos ter cuidado ao colocar essas pessoas para trabalharem juntas. Nos leva também a prever conflitos na agência do SAC, quando um grupo de funcionários do segundo mapa está sendo supervisionado por pessoas que estão acostumadas a tratar com funcionários do primeiro mapa e que, provavelmente, já foram funcionários do primeiro mapa.

A diferença de focos principais nos dois mapas, o primeiro no "perito técnico de polícia" e o segundo no "cidadão", explica problemas identificados e posteriormente confirmados pelos gerentes dessas organizações. Situações em que o atendente da PostData quer emitir a carteira de identidade e o perito não pode permitir por qualquer irregularidade mínima nos documentos necessários.

Ficou evidenciado também o receio que os funcionários terceirizados apresentam com relação a realização da atividade, uma vez que não são peritos e não possuem a mesma capacidade destes para reconhecer um documento irregular ou algo que possa comprometer a segurança da confecção das carteiras. Isso reflete também como os conceitos formulados por esses funcionários sofrem influência daqueles formulados pelos funcionários públicos, já que estes estão em constante interação.

É importante salientar que algumas conclusões poderiam ser tiradas a partir das entrevistas e dos textos, porém, no momento em que construímos os mapas, as informações se tornam mais compreensíveis e passam a fazer mais sentido, pois, a partir da representação dos conceitos em mapas, podemos visualizar a lógica de pensamento daqueles que foram entrevistados e até entender como estes costumam agir. E, especialmente quando se trata de comparar informações, torna-se mais fácil fazê-lo a partir de dois mapas, do que de dois textos ou de seis entrevistas.

Com a realização deste trabalho, podemos concluir que resultados podem ser obtidos para a análise de interorganizações através da comparação entre mapas cognitivos que representam as organizações envolvidas. Sendo assim, fica fácil reconhecer a utilidade dos mapas cognitivos como ferramenta de pesquisa para os Estudos Organizacionais.

Referências:

BASTOS, A. V. B. Mapas cognitivos: ferramentas de pesquisa e intervenção em processos organizacionais. Anais: Encontro Nacional de Estudos Organizacionais, 2000 .

Organização e cognição; o que emerge desta enterface? PINA, M., RODRIGUES, S. B. (org). Estudos organizacionais: novas perspectivas. Lisboa, 2000. 
--------- Organizações como "corpos de pensamento e ação": o olhar cognitivista e suas implicações para a gestão das pessoas. DAVEL, E., VERGARA, S. (org). Gestão de pessoas e subjetividade: dos recursos aos seres humanos nas empresas contemporâneas. Petrópolis, 2000.

BOUGON, M. Uncovering cognitives maps: the Self-Q technique. In: MORGAN, G. Beyond Method. Newbury Park, SAGE, 1983.

BOUGON, M., WEICK, K., BINKHORST, D. Cognition in organizations: an analysis of Utrecht Jazz Orchestra. Administrative Science Quartely, vol 22, pp.606$631,1977$.

DOWNEY, H. K., e BRIEF, A. P. How cognitive structures affect organizational design. Em: D. A. GIOIA e H. P. SIMS Jr, The Thinking Organization. San Francisco, CA: Jossey-Bass. Inc: Publishers, pp.165-190, 1986.

HARD, C., PHILIPPS, N. Strategies of engagement; lessons from the critical examinations of collaboratoin and conflict in an interorganizational domain. Organization Sciencen, vol 9, nº 2, 1998.

HUFF, A. S. Mapping Strategic Thought. Em A. S. HUFF (Ed). Mapping Strategic Thought. Chichester: Wiley, pp.11-49, 1990.

LAUKKANEN, M. Comparative cause mapping of organizational cognitions. Organization Science, vol 5, no 3, 1994.

NICOLINI, D. Comparing methods for mapping organizational cognition. In: Organizations Studies. EGOS, London, 1999.

POESI, F. Analyses dés representations et dés discours. In: THIETART, R. Mèthodes de Recherche et Management. Paris, Dunod, 1999.

PORAC, J. F., MEINDL, J. R., STUBBART, C. Introduction. Em, J. PORAC, J. MEINDL, C. STUBBART (eds) Cognition within and between organizations . Thousand Oaks, CA:Sage, 1996.

WEICK, K. A Psicologia Social da Organização. Tradução: Dante Moreira Leite. Editora Edgard Blücher Ltda. São Paulo, 1973. 


\footnotetext{
${ }^{\text {i }}$ Para melhor compreensão deste trabalho o termo agência será usado para denominar os pontos dos serviços de identificação localizados dentro dos postos do SAC. Pois cada posto do SAC possui diversas agências de serviços diferenciados.

${ }^{\text {ii }}$ Mente aqui pode ser entendida como entidade interna, autônoma, com faculdades mentais controladoras das ações, pensamentos, sentimentos, vontades e decisões das pessoas (BASTOS,2000).

iii De acordo com BASTOS (2000), a analogia entre o funcionamento da mente humana e o computador apóia-se no princípio de que ambos são sistemas cognitivos, funcionalmente equivalentes, já que processam informações através da manipulação de símbolos.
} 